\title{
The perioperative application of continuous cerebral autoregulation monitoring for cerebral protection in elderly patients
}

\author{
Yunliang Zhang ${ }^{1 \#}$, Jian Tan ${ }^{2 \#}$, Peiyao Li $^{3}$, Xiaoying Zhang ${ }^{1}$, Yitian Yang ${ }^{1}$, Yanhong Liu ${ }^{1}$, Qiang Fu ${ }^{1}$, \\ Jiangbei $\mathrm{Cao}^{1}$, Weidong $\mathrm{Mi}^{1}$, Hong $\mathrm{Zhang}^{1}$, Hao $\mathrm{Li}^{1}$ \\ ${ }^{1}$ Department of Anesthesiology, The First Medical Center, Chinese PLA General Hospital, Beijing, China; ${ }^{2}$ Department of Thoracic Surgery, the \\ Seventh Medical Center, Chinese PLA General Hospital, Beijing, China; ${ }^{3}$ Global Health Drug Discovery Institute, Beijing, China \\ Contributions: (I) Conception and design: H Li, Y Zhang, J Tan; (II) Administrative support: H Zhang, W Mi, Q Fu, J Cao; (III) Provision of study \\ materials or patients: Y Liu, X Zhang; (IV) Collection and assembly of data: Y Yang; (V) Data analysis and interpretation: P Li, Y Zhang; (VI) \\ Manuscript writing: All authors; (VII) Final approval of manuscript: All authors. \\ "These two authors contributed equally to this work. \\ Correspondence to: Hao Li; Hong Zhang. Department of Anesthesiology, The First Medical Center, Chinese PLA General Hospital, Beijing 100853, \\ China. Email: lihao301@126.com; mazuimao301@163.com.
}

Background: The majority of surgical patients aged 65 years and over are accompanied with underlying conditions, making them susceptible to perioperative cerebral complications. Here, we investigated the clinical value of continuous cerebral autoregulation (CA) monitoring in protecting against cerebral dysfunction in elderly patients undergoing surgery.

Methods: This study enrolled 40 elderly patients (aged $\geq 65$ years) and 40 middle-aged patients (aged 45 to 64 years) selected to undergo robotic-assisted laparoscopic radical prostatectomy. Cerebral oxygenation was assessed by regional cerebral oxygen saturation $\left(\mathrm{rScO}_{2}\right)$ using near-infrared spectroscopy (NIRS). CA function was estimated using the cerebral oximetry index $\left(\mathrm{CO}_{\mathrm{X}}\right)$, which is the rolling correlation between $\mathrm{rScO}_{2}$ and the mean arterial pressure (MAP). With the patient in the Trendelenburg position, the $\mathrm{rScO}_{2}, \mathrm{MAP}$, calculated $\mathrm{CO}_{\mathrm{x}}, \mathrm{HR}$, end-tidal $\mathrm{CO}_{2}$, and sevoflurane concentrations were continuously recorded. Standardized anesthesia was administered to all patients (sevoflurane, propofol, remifentanil, and rocuronium). Postoperative delirium (POD) was screened for daily using the Confusion Assessment Method (CAM). The primary outcome was the difference in periods of CA dysfunction between the elderly and middle-aged groups. Secondary outcomes included the incidence of POD and the optimal MAP range in the 2 groups.

Results: Taking positive $\mathrm{CO}_{\mathrm{x}}$ values (cutoff $\geq 0.3$ ) to reflect periods of CA dysfunction, we found that the cumulative duration of CA dysfunction in the Trendelenburg position was longer in elderly patients than in middle-aged patients [ratio of cumulative time of CA dysfunction: middle-aged group, 32.8\% $(26.3 \%$, $43.1 \%)$ vs. elderly group, $42.2 \%(33.1 \%, 51.2 \%)](\mathrm{P}<0.01)$, which showed that CA function was less efficient in elderly patients. Three patients $(7.5 \%)$ in the elderly group and 1 patient $(2.5 \%)$ in the middle-aged group screened positive for POD on at least 1 day during their hospital stay. Additionally, using the $\mathrm{CO}_{\mathrm{x}^{-}}$ based method, we estimated the optimal MAP targets in the middle-aged and elderly groups to be (67.8 \pm 8.9 , $116.4 \pm 10.5)$ and $(71.2 \pm 12.5,111.3 \pm 8.9) \mathrm{mmHg}$, respectively.

Conclusions: The brains of patients $\geq 65$ years are more vulnerable to systemic insult compared with those of middle-aged patients. POD may be associated with CA dysfunction. NIRS-derived $\mathrm{CO}_{\mathrm{X}}$ can be used to identify the optimal MAP range.

Keywords: Cerebral autoregulation (CA); regional cerebral oxygen saturation $\left(\mathrm{rScO}_{2}\right)$; optimal mean arterial pressure (optimal MAP); robotic-assisted laparoscopic radical prostatectomy (RALP) 
Submitted Jan 31, 2021. Accepted for publication Apr 21, 2021.

doi: 10.21037/apm-21-707

View this article at: http://dx.doi.org/10.21037/apm-21-707

\section{Introduction}

Robotic-assisted laparoscopic urinary tract surgery requires the patient to be kept in the Trendelenburg position for a long time, as well as $\mathrm{CO}_{2}$ pneumoperitoneum (1). These conditions impact the patient's cerebral hemodynamics tremendously and may lead to increased cerebral blood volume, intracranial hypertension, dysfunctional cerebral autoregulation (DCA), and changes in cerebral oxygen metabolism (2). Robotic-assisted laparoscopic urinary tract surgery is mostly applied to treat elderly patients, a large proportion of whom are accompanied with underlying diseases, increased systemic vascular fragility, decreased cerebral blood flow, brain tissue degradation, impaired cerebral autoregulation (CA) capacity, and increased risks of postoperative stroke, postoperative delirium (POD), and other brain dysfunctions (3). Delirium is fairly common in elderly patients following surgery, and refers to an acute onset of changes in cognitive function, which manifest as decreased consciousness, inattention, and confusion over time. POD often starts in the recovery room and typically occurs within 5 days after surgery (4). The incidence of POD among elderly patients undergoing major abdominal surgery has been reported to reach as high as $38 \%$ (5). Patients with POD have longer hospital stays, higher medical costs, more postoperative complications (including perioperative neurocognitive impairment), and an increased risk of death (6).

The mechanism underlying delirium is unclear. Some studies involving severe cardiac surgery, traumatic brain surgery, and intensive care unit (ICU) patients have shown DCA to be closely related to the occurrence of POD (7). The present study included 40 middle-aged patients (8) and 40 elderly patients undergoing robotic-assisted laparoscopic prostatectomy (RALP) for prostate cancer under general anesthesia. Cerebral autoregulation monitoring has been used widely in clinical practice and is associated with better outcomes especially in traumatic brain injury (9-11). But similar study focused on elderly patients is few especially those with high risk of delirium. Near-infrared spectroscopy (NIRS) was used to continuously monitor regional cerebral oxygen saturation $\left(\mathrm{rScO}_{2}\right)$, and the mean arterial pressure (MAP) was synchronously collected (12). The correlation between $\mathrm{rScO}_{2}$ and the MAP was analyzed to obtain the dynamic cerebral oximetry index $\left(\mathrm{CO}_{\mathrm{X}}\right)$ as a reflection of CA. A $\mathrm{CO}_{\mathrm{X}}$ of $\geq 0.3$ is defined as CA impairment. This study made the assumption that under general anesthesia, elderly patients would show a higher degree of CA impairment than middle-aged patients, thus elevating the risk of POD. With the use of $U$-shaped diagram fitted to the $\mathrm{CO}_{\mathrm{X}}$ curve, we aimed to retrospectively identify the optimal intraoperative MAP and blood pressure range when the CA function was optimal. We present the following article in accordance with the TREND reporting checklist (available at http://dx.doi.org/10.21037/apm-21-707).

\section{Methods}

\section{Study population}

This is a prospective observational study that sequentially included patients who were admitted to the First Medical Centre of the PLA General Hospital from April to October 2019 and received RALP for prostate cancer under general anesthesia. The patients were grouped by age: 40 patients aged 45 to 64 years were enrolled as the middle-aged group (8), and 40 patients aged $\geq 65$ years were enrolled as the elderly group. The exclusion criteria for enrollment were: (I) American Society of Anaesthesiologists (ASA) grade IV or above; (II) severe visual or hearing impairment; (III) brain trauma, cerebrovascular disease, mental disorders, endocrine or metabolic disease, severe heart or lung disease, liver or kidney insufficiency, or severe malnutrition before surgery; (IV) recent alcohol abuse or insomnia; (V) long-term use of psychotropic drugs; and (VI) a mini-mental state examination (MMSE) score $<24$ points. Exclusion criteria after enrollment included: (I) intraoperative $\mathrm{P}_{\mathrm{ET}} \mathrm{CO}_{2}$ (end-tidal carbon dioxide partial pressure) $>45 \mathrm{mmHg}$ and (II) intraoperative MAP cannot be maintained within $20 \%$ over or under the baseline value of admission. This study was reviewed and approved by the Ethics Committee of the PLA General Hospital (No. S2019-120-02). A signed informed consent form was obtained from each patient or from a member of their family. All procedures performed in this study involving human participants were in accordance with the 
Declaration of Helsinki (as revised in 2013).

\section{Sample size calculation}

The main indicator in this study was the automatic adjustment of cerebral blood flow. Based on previous studies, a difference in $\mathrm{CO}_{\mathrm{X}}$ value of 0.15 between the 2 groups was considered to show clinical significance. It was estimated that the sample size would require at least 32 patients per group to reach $90 \%$ of the inspection level $(\alpha=0.05)$ (9). Taking into account the lack of $\mathrm{CO}_{\mathrm{X}}$ data during surgery, the failure of monitoring, and loss to followup, we decided to increase the sample size by $20 \%$; thus, 40 patients were included in each group.

\section{Anesthesia}

The methods of anesthesia were the same in the 2 groups. In the operating room, the patient's heart rate was monitored by electrocardiogram (ECG), and their noninvasive blood pressure (NIBP), oxygen saturation $\left(\mathrm{SpO}_{2}\right)$, and bispectral index (BIS) were also routinely monitored. A NIRS machine was used to record $\mathrm{rScO}_{2}$ (INVOS 5100c, Covidien, USA) according to the equipment protocol.

\section{Induction of anesthesia}

After the establishment of the intravenous channel, patients were administered oxygen via a face mask, and sequentially injected with midazolam $0.06 \mathrm{mg} / \mathrm{kg}$, sufentanil $0.3 \mu \mathrm{g} / \mathrm{kg}$, propofol $2 \mathrm{mg} / \mathrm{kg}$, and rocuronium $0.6 \mathrm{mg} / \mathrm{kg}$. The surgical conditions were: intratracheal intubation, mechanically controlled ventilation, tidal volume $6-8 \mathrm{~mL} / \mathrm{kg}$, respiratory rate $12-15$ times/min, inhalation-expiration ratio $1: 2$, fresh oxygen flow rate $2 \mathrm{~L} / \mathrm{min}$. After anesthesia induction, the inhaled oxygen concentration was adjusted to $60 \%$, and the breathing parameters were adjusted to maintain $\mathrm{P}_{\mathrm{ET}} \mathrm{CO}_{2}$ at $35-45 \mathrm{mmHg}$. The left radial artery was punctured for the analysis of invasive arterial pressure and arterial blood gas. The nasopharyngeal temperature was monitored, and a heating device was employed to maintain the nasal temperature between 36 and $37^{\circ} \mathrm{C}$.

\section{Maintenance of anesthesia}

Anesthesia was maintained by routine intravenousinhalational anesthesia. After induction, propofol 4$8 \mathrm{mg} /(\mathrm{kg} \cdot \mathrm{h})$ and remifentanil $0.1-0.2 \mu \mathrm{g} /(\mathrm{kg} \cdot \mathrm{min})$ were injected, and inhaled sevoflurane $(<1$ minimum alveolar concentration) was administered continuously; sufentanil and rocuronium were added in order to maintain analgesia and muscle relaxation. The real-time end-tidal sevoflurane concentration $\left(\mathrm{ET}_{\mathrm{SEV}}\right)$ was recorded. After establishing $12 \mathrm{mmHg}(1 \mathrm{mmHg}=0.133 \mathrm{kPa})$ pneumoperitoneal pressure according to surgical requirements, we tilted the shaker into the Trendelenburg position (head low + foot high + lithotomy position). During the operation, the MAP was maintained within $\pm 20 \%$ of the baseline value; the depth of anesthesia was adjusted to maintain a BIS value of 40-60; and colloidal fluid was administered or concentrated red blood cells were infused according to bleeding and circulatory fluctuations in order to maintain hematocrit $($ HCT $)>24 \%$.

\section{Postoperative analgesia and follow-up}

Sufentanil $10 \mu \mathrm{g}$ (for elderly and frail patients, an appropriate dose reduction was made, and non-steroidal analgesics could also be used) and ondansetron $8 \mathrm{mg}$ were administered 30 minutes before skin suture. After the operation, the patients were hooked up to a sufentanil 2 $6 \mu \mathrm{g} / \mathrm{h}$ intravenous analgesia pump, and the numerical rating scale (NRS) pain score was maintained at $\leq 3$ points. On postoperative days 1 to 5 , an anesthesiologist or anesthesia nurse who was not involved in the anesthesia procedure and was familiar with the diagnostic criteria of POD assessed the patients for delirium using the Confusion Assessment Method (CAM).

\section{Blood sample collection and testing}

Radial artery blood was collected before and after surgery to assay arterial blood gas (BD arterial lancet, USA), and venous blood was collected for the analysis of the brain injury biomarkers serum $S 100 \beta$ protein, neuron-specific enolase (NSE), and interleukin (IL)-6 (Proteintech kit, Wuhan Sanying, China).

\section{Direct observation indicators}

The NIRS-monitored $\mathrm{rScO}_{2}$ was collected every 6 seconds, and the 2 -sided average value was taken. The invasive MAP, $\mathrm{HR}, \mathrm{P}_{\mathrm{ET}} \mathrm{CO}_{2}$, and $\mathrm{ET}_{\mathrm{SEV}}$ were continuously recorded while the patient was in the Trendelenburg position. Pre- and postoperative changes in arterial blood gas and brain injury biomarkers were observed, as was the occurrence of POD. 

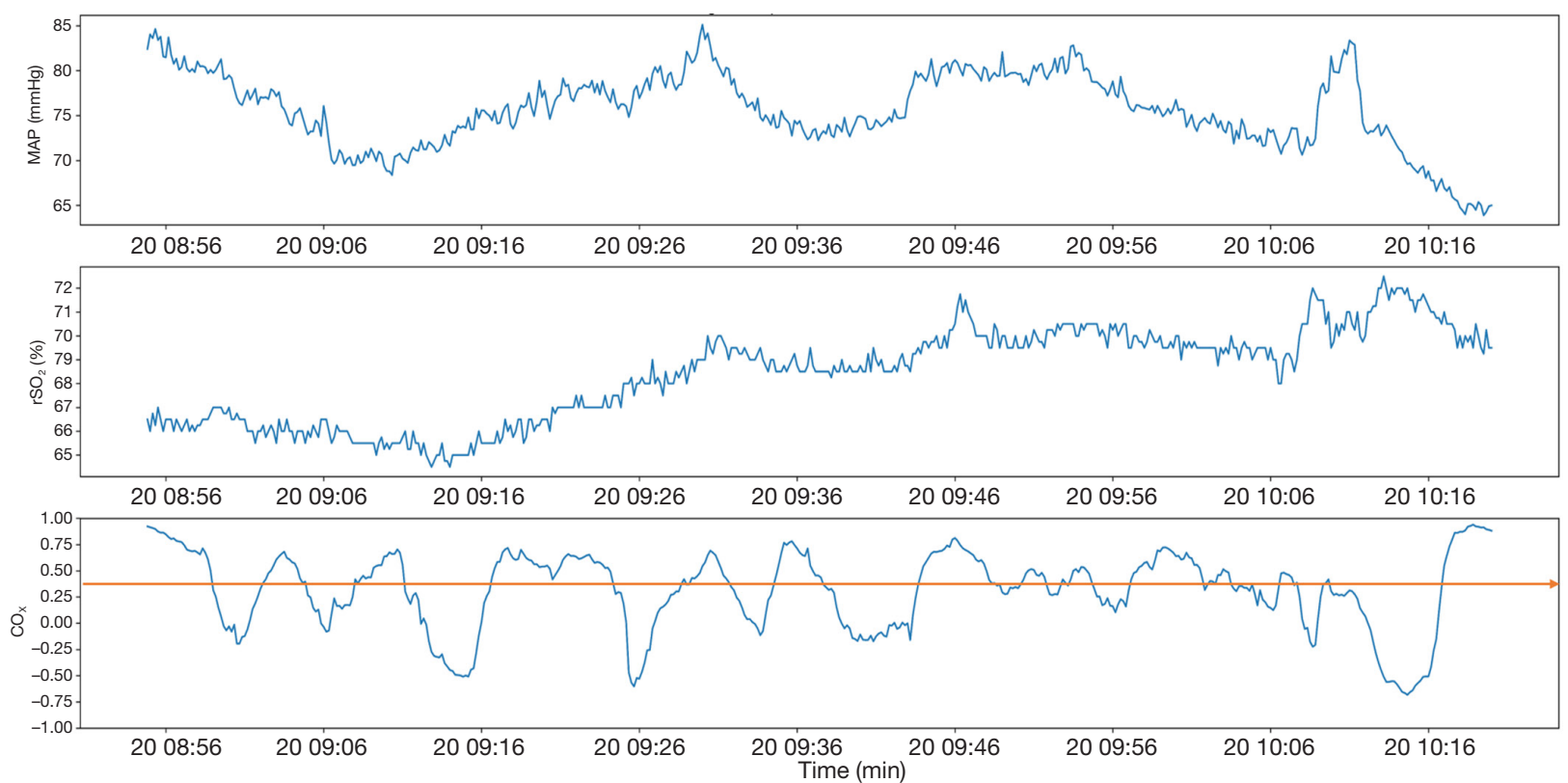

Figure $1 \mathrm{MAP}$ data (top), $\mathrm{rScO}_{2}$ data (middle), and the $\mathrm{CO}_{\mathrm{x}}$ curve with a time window of 5 minutes (bottom). The curve above the red line represents DCA. MAP, mean arterial pressure; $\mathrm{rScO}_{2}$, regional cerebral oxygen saturation; $\mathrm{CO}_{\mathrm{x}}$, cerebral oximetry index; DCA, dysfunctional cerebral autoregulation. X-axis stand for time points: 20 represents the date we took these data; the time after " 20 " means the exact time point on that day and the minimal interval between two time points is 10 minutes.

$\mathrm{CO}_{\mathrm{X}}$

Using MATLAB software (version R2017b, MathWorks, USA), we wrote a program to obtain $\mathrm{CO}_{\mathrm{X}}$. With the patient in the Trendelenburg position, the $\mathrm{rScO}_{2}$ and synchronously recorded invasive MAP data were collected every 6 seconds, and the moving time window was set to 5 minutes. A total of 30 sets of data were obtained, and Spearman's correlation coefficient was used to continuously analyze $\mathrm{CO}_{\mathrm{X}}$ changes over time. As the relationship between $\mathrm{rScO}_{2}$ and MAP was not necessarily linear, Spearman's correlation coefficient was applied instead of Pearson's correlation coefficient. At the same time, in order to find the best $\mathrm{CO}_{\mathrm{X}}$ curve, the $\mathrm{CO}_{\mathrm{X}}$ curve was optimized on the time window, and various time windows $(1,5,10,15,20,25$, and 30 minutes) were selected to observe the change trend of $\mathrm{CO}_{\mathrm{X}} \cdot \mathrm{CO}_{\mathrm{X}} \geq 0.3$ was defined as DCA (Figure 1). CA impairment in the 2 groups was represented by the ratio of DCA cumulative time (\%), which was calculated as the difference between the cumulative time of $\mathrm{CO}_{\mathrm{x}}$ and the threshold divided by the total recording time in the Trendelenburg position, multiplied by 100 [DCA cumulative time ratio (\%) = (cumulative time greater than the threshold/head-down duration) $\times 100 \%$.

\section{Intraoperative optimal MAP and blood pressure range}

According to the approach commonly used in the literature (9), with MAP as the abscissa and $5 \mathrm{mmHg}$ as a grid, the U-shaped curve was fitted to the $\mathrm{CO}_{\mathrm{X}}$ value obtained from the length of each time window in the ordinate, and the lowest point of the curve (the optimal MAP is related to the lowest $\mathrm{CO}_{\mathrm{X}}$ ) was considered to be the optimal intraoperative MAP $\left(\mathrm{MAP}_{\mathrm{OPT}}\right)$. A horizontal line was drawn with the $\mathrm{CO}_{\mathrm{X}}$ threshold as $\geq 0.3$, which represents the shift from normal CA to dysfunction. The blood pressure range corresponding to the intersection with the curve is called the optimal blood pressure range $\left(\mathrm{MAP}_{\mathrm{OPT}}\right.$ range) (Figure 2).

\section{Statistical analysis}

Statistical analyses were performed using SPSS 22.0 (IBM, Chicago, USA) statistical software. Continuous measurement data with a normal distribution were expressed as mean \pm standard deviation $\left(\bar{x}_{ \pm \mathrm{S}}\right)$, and measurement data not conforming to a normal distribution were expressed as mean $(\mathrm{Q} 1, \mathrm{Q} 3)$. Categorical variables were expressed as the number of cases (n, \%). The correlation of $\mathrm{rScO}_{2}$ with the 


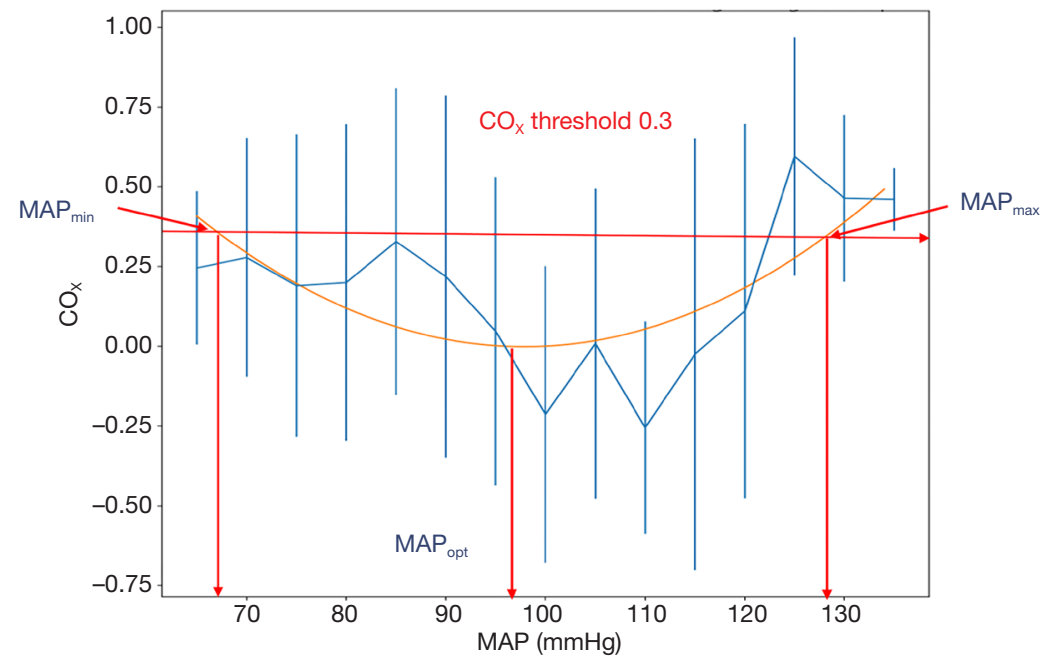

Figure 2 Optimal mean arterial pressure and blood pressure range. A horizontal line was drawn with the $\mathrm{CO}_{\mathrm{X}}$ threshold as $\geq 0.3$, which represents the shift from normal CA to dysfunction. The blood pressure range corresponding to the intersection with the curve is called the optimal blood pressure range $\left(\mathrm{MAP}_{\mathrm{OPT}}\right.$ range).

MAP was analyzed using Spearman's correlation coefficient. Statistical differences between groups were analyzed using Fisher's test for classification data in a $2 \times 2$ contingency table or $t$-test for comparison between groups. The Wilcoxon rank-sum test was used to compare continuous data. $\mathrm{P}<0.05$ indicated a statistically significant difference.

\section{Results}

\section{Baseline characteristics}

During the study period, 122 patients (54 patients aged $45-64$ years old and 68 patients aged $\geq 65$ years old) were sequentially enrolled. After the exclusion of 42 cases who did not meet the exclusion criteria or did not meet the research requirements during surgery, 80 patients were finally enrolled in the analysis (40 in the elderly group and 40 in the middle-aged group). All patients in the study had ASA classifications of grade I to III. Table 1 shows the patients' general conditions, along with their intraoperative and recovery status. None of the patients received a blood transfusion during surgery. No significant difference was found in body mass index, blood loss, fluid intake, or the time spent in the head-down position between the 2 groups $(\mathrm{P}>0.05)$; however, the recovery time in the elderly group was significantly longer than that in the middle-aged group $(\mathrm{P}<0.05)$. There were no clinically relevant changes in $\mathrm{ET}_{\mathrm{SEV}}$ or $\mathrm{SpO}_{2}$ over time in either of the groups. Preoperative and postoperative blood gas analysis of the elderly group revealed the difference between the average values of $\mathrm{P}_{\mathrm{ET}} \mathrm{CO}_{2}$ and $\mathrm{PaCO}_{2}$ to be $(3.4 \pm 3.1)$ and $(3.7 \pm 3.6) \mathrm{mmHg}$, respectively; in the middleage group, the difference between the average values of $\mathrm{P}_{\mathrm{ET}} \mathrm{CO}_{2}$ and $\mathrm{PaCO}_{2}$ was $(3.1 \pm 3.2)$ and $(3.5 \pm 3.2) \mathrm{mmHg}$, respectively.

\section{Time window}

The $\mathrm{CO}_{\mathrm{X}}$ is a rolling coefficient of the correlation between the MAP and $\mathrm{rScO}_{2}$, which reflects CA. In this study, to optimize the detection of DCA, different moving time window lengths (1-30 minutes) were selected to generate the $\mathrm{CO}_{\mathrm{X}}$ (Figure 3). A moving time window that is too short (e.g., $<1 \mathrm{~min}$ ) will generate a noisy $\mathrm{CO}_{\mathrm{X}}$ signal, resulting in the signal being indistinguishable between different patients. A medium-length time window (5-10 minutes) can better reveal the $\mathrm{CO}_{\mathrm{X}}$ characteristics of the time series between different patients. The selection of a longer time window will make the $\mathrm{CO}_{\mathrm{X}}$ signal smoother and reduce the $\mathrm{CO}_{\mathrm{X}}$ dynamic changes detected in the medium time window. In this study, we ultimately selected a mediumlength time window, and normal CA and intermittent DCA could be observed in patients during their entire time in the Trendelenburg position. Sensitivity analysis based on parameter changes showed that the best time window length for identifying DCA was 5 minutes. 
Table 1 Comparison of the general conditions of the patients in the 2 groups

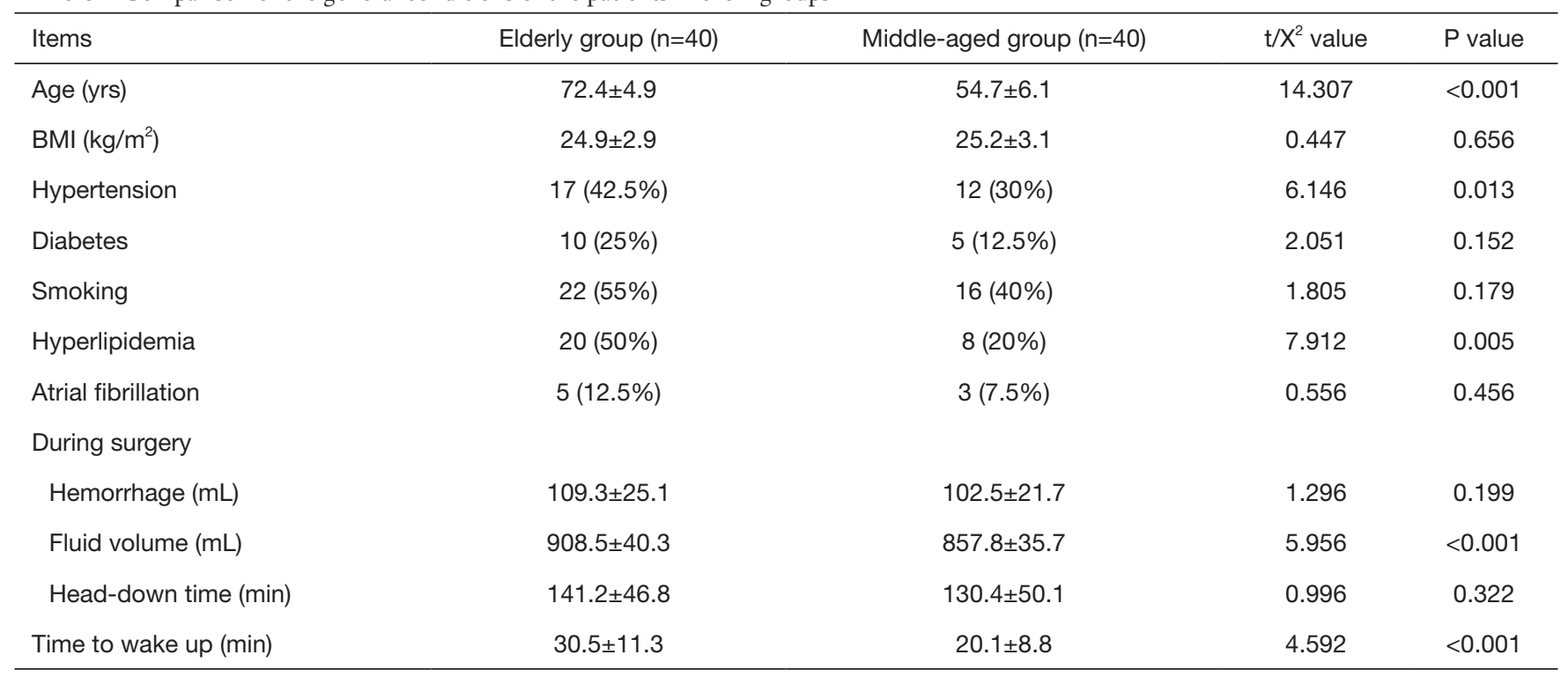

\section{Intraoperative occurrence of DCA and POD in the 2 groups}

Regarding the major observation indicator, all patients in the study showed a certain degree of DCA, with the cumulative time of DCA in the elderly group being significantly longer than that in the middle-aged group (Table 2). In the elderly and middle-aged groups, 3 cases $(7.5 \%)$ and 1 case $(2.5 \%)$ of POD were screened out, respectively, during follow-up; all of these patients experienced delirium for at least 1 day during hospitalization. Also, of the 42 patients excluded from the study, 2 (4.8\%) patients in the elderly group developed delirium. Both of them had high $\mathrm{P}_{\mathrm{ET}} \mathrm{CO}_{2}$ and subcutaneous emphysema during the operation, and blood gas analysis showed a low partial pressure of oxygen. After surgery, they were finally sent to the ICU without extubation. These 5 elderly patients were all $>70$ years old, and the average head-down time exceeded 3 hours.

\section{Intraoperative optimal MAP and blood pressure range}

In this study, a moving time window of 5 minutes was selected to obtain the $\mathrm{MAP}_{\mathrm{OPT}}$. After fitting, the $\mathrm{CO}_{\mathrm{X}}$ curve for 25 cases in the middle-aged group and 23 cases in the elderly group exhibited a typical U-shape. The intraoperative $\mathrm{MAP}_{\text {OPT }}$ was found to be $83.6 \pm 6.8$ and $90.9 \pm 6.1 \mathrm{mmHg}$ in the middle-aged group and the elderly group, respectively (Figure 4). A horizontal line was drawn with a $\mathrm{CO}_{\mathrm{X}}$ threshold of 0.3 , and the optimal blood pressure ranges of the middle-aged and elderly patients were shown to be $(67.8 \pm 8.9 ; 116.4 \pm 10.5)$ and $(71.2 \pm 12.5 ; 111.3 \pm 8.9)$ mmHg, with an obviously narrowed blood pressure adjustment range in the elderly group $(\mathrm{P}<0.01)$ (Table 3$)$.

\section{Changes in brain injury biomarkers}

No significant changes were observed in the levels of serum S100 $\beta$ protein, NSE, and IL-6 before and after surgery in either group (Table 4).

\section{Discussion}

This study optimized the length of the moving window for $\mathrm{CO}_{\mathrm{X}}$ generation. Selecting a time window of 5 minutes can increase the sensitivity of DCA detection. We found that CA was significantly more impaired in elderly patients than in middle-aged patients when in the Trendelenburg position $(\mathrm{P}<0.01)$. This study demonstrates that under the conditions of a strict head-down posture and pneumoperitoneum, the cumulative time of DCA in elderly patients during surgery was longer than that in middle-aged patients, which may contribute to POD.

NIRS can penetrate the thickness of the skull to distinguish the difference in the absorption spectra of oxyhemoglobin and deoxyhemoglobin in intracranial brain tissue, thereby obtaining $\mathrm{rScO}_{2}$. The $\mathrm{rScO}_{2}$ value reflects the balance of oxygen supply and demand, and 

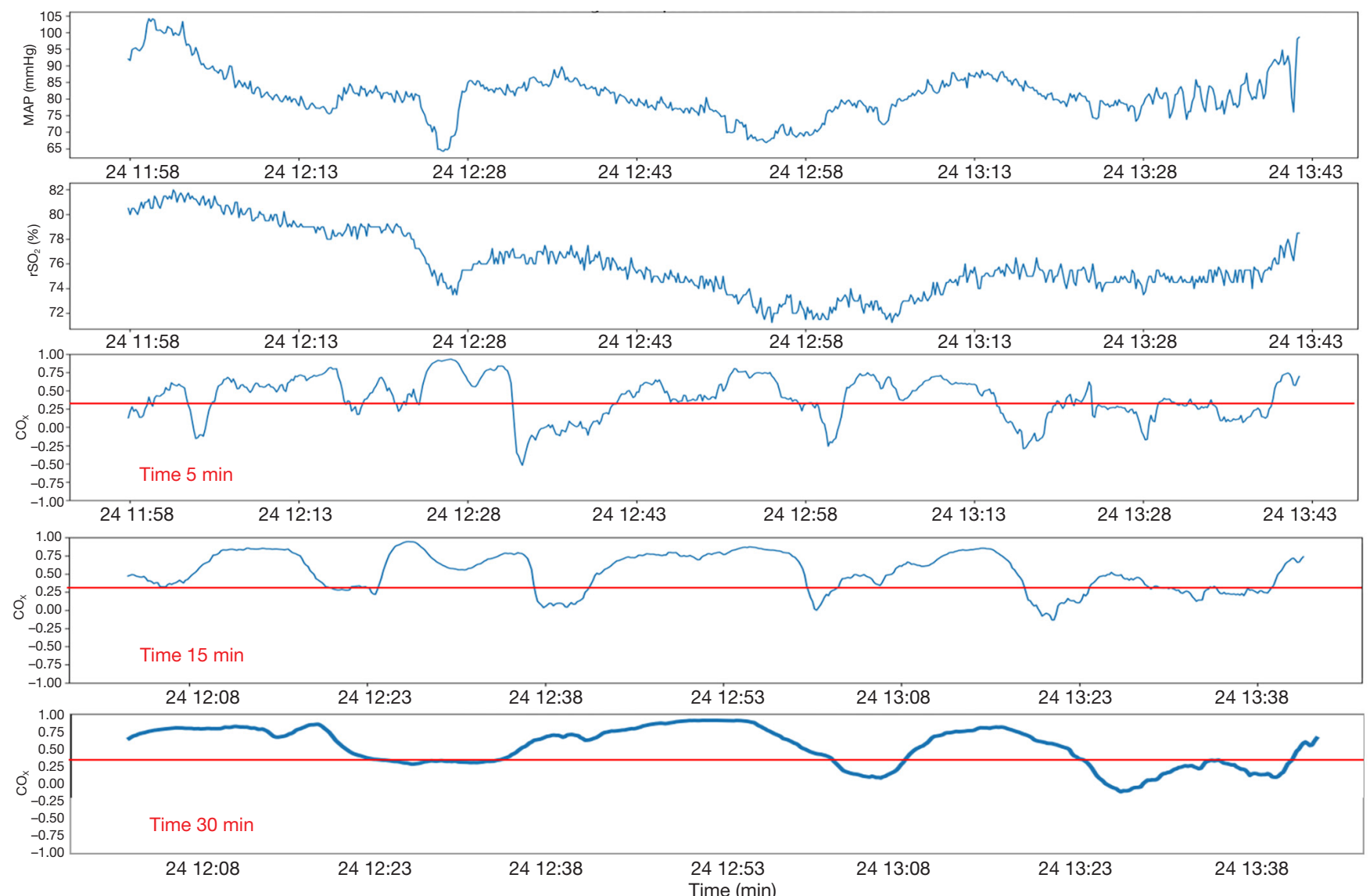

Figure 3 Time window selection (for the same patient, the $\mathrm{CO}_{\mathrm{x}}$ time windows are 5, 15, and 30 minutes, respectively, and the red line represents the $\mathrm{CO}_{\mathrm{X}}$ threshold of 0.3 ). To optimize the detection of DCA, different moving time window lengths (1-30 minutes) were selected to generate the $\mathrm{CO}_{\mathrm{X}}$. A moving time window that is too short (e.g., $<1 \mathrm{~min}$ ) will generate a noisy $\mathrm{CO}_{\mathrm{X}}$ signal, resulting in the signal being indistinguishable between different patients. $\mathrm{CO}_{\mathrm{X}}$, cerebral oximetry index.

Table 2 Comparison of intraoperative DCA between the 2 groups

\begin{tabular}{|c|c|c|c|c|}
\hline Items & Elderly group $(n=40)$ & Middle-aged group $(n=40)$ & $t / X^{2}$ value & $P$ value \\
\hline DCA time (s) & $3,554.2 \pm 376.8$ & $2,799.9 \pm 309.2$ & 7.748 & $<0.001$ \\
\hline DCA-CTR (\%, median, IQR) & $42.2(33.1,51.2)$ & $32.8(26.3,43.1)$ & & $<0.001$ \\
\hline
\end{tabular}

DCA-CTR, cumulative time ratio of DCA; DCA, dysfunctional cerebral autoregulation; IQR, interquartile range.

indirectly reflects cerebral blood flow. The many benefits of NIRS include its non-invasive, real-time, continuous, easy operation, and bedside monitoring (11). Some studies have failed to explain the complex relationship between low intraoperative $\mathrm{rScO}_{2}$ values and POD $(13,14)$. This failure may be attributable to the large interindividual differences in the basic value of $\mathrm{rScO}_{2}$ resulting from various preoperative pathophysiological factors, and may be also relevant to the definition of deoxgenation and the duration of intraoperative hypoxia (15). In their study, Goettel et al. (16) explored the relationship between CA, cerebral oxygenation, the brain injury marker NSE, 

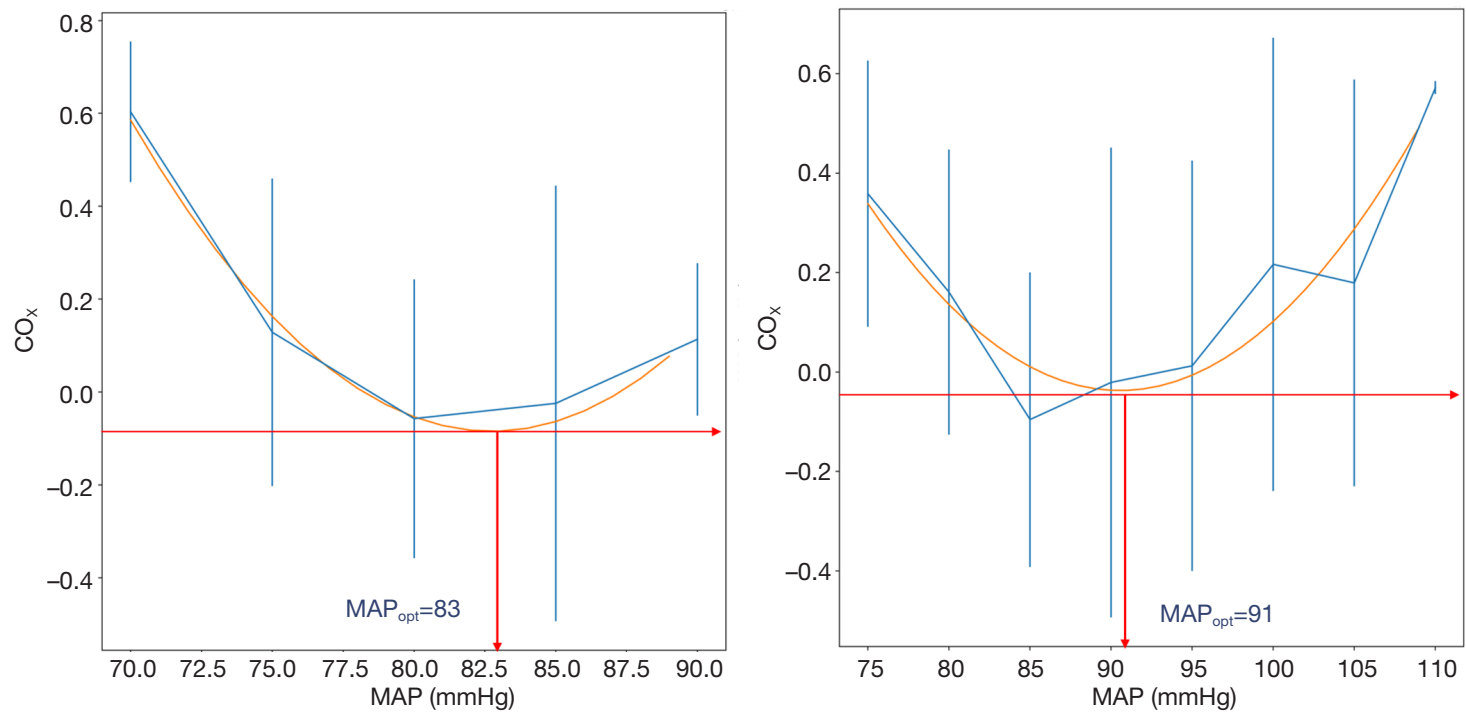

Figure 4 Optimal mean arterial pressure during operation in middle-aged and elderly patients. The red line stand for the $\mathrm{CO}_{\mathrm{x}}$ curve for 25 cases in the middle-aged group (left) and 23 cases in the elderly group (right) exhibited a typical U-shape.

Table 3 The optimal blood pressure range of the two groups of patients during operation

\begin{tabular}{|c|c|c|c|c|}
\hline Blood pressure & Elderly group $(n=40)$ & Middle-aged group $(n=40)$ & $\mathrm{t} / \mathrm{X}^{2}$ value & $P$ value \\
\hline LL of BP $(\mathrm{mmHg})$ & $71.2 \pm 12.5$ & $67.8 \pm 8.9$ & 1.401 & 0.165 \\
\hline Difference (mmHg) & $40.1 \pm 7.9$ & $48.6 \pm 10.3$ & 4.141 & $<0.001$ \\
\hline
\end{tabular}

UL of BP, upper limit of blood pressure; LL of BP, lower limit of blood pressure.

Table 4 Comparison of brain injury biomarkers in the 2 groups

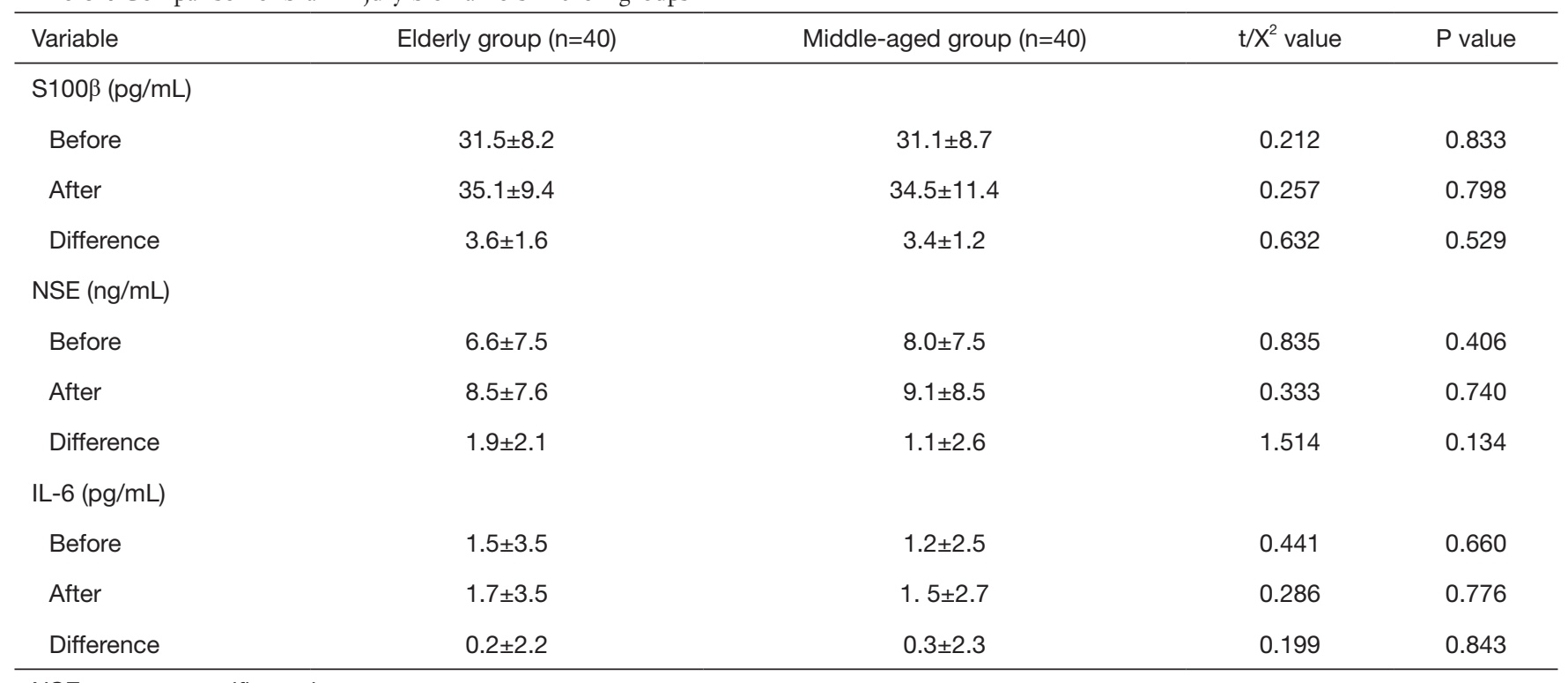

NSE, neuron-specific enolase. 
and postoperative cognitive dysfunction (POCD) in 82 elderly patients undergoing non-cardiac major surgery under general anesthesia. The impairment of CA during surgery were revealed to be closely correlated with POCD. Furthermore, an increase in C-reactive protein (CRP) on the $2^{\text {nd }}$ day post surgery and the highest postoperative serum NSE value were also found to be related to the occurrence of POCD; however, there was no obvious correlation between brain oxygenation and POCD.

Under different physiological conditions, the resistance blood vessels in the brain contract or relax in response to blood pressure fluctuations to maintain a relatively constant cerebral blood flow, thereby meeting the brain's metabolic needs. This process is called CA, which is a mechanism to protect the stability of cerebral blood flow. Elderly patients undergoing surgery are often accompanied with a variety of comorbidities. In elderly patients, the fragility of the cerebral blood vessels increases, the ability to regulate cerebral blood flow may be impaired, and the adjustment range is narrowed. When the MAP is lower than the limit of CA, cerebral ischemia events occur (17). When the MAP is above the autoregulation range, overperfusion of the brain can trigger cerebral edema or hemorrhage (18). Previous studies on patients undergoing cardiac or brain trauma surgery, and critically ill patients in the ICU have uncovered a close relationship between DCA and a poor prognosis. Lee et al. (19) studied 40 middle-aged patients with respiratory failure or shock who were admitted to the ICU and found that the early 72-hour accumulation of DCA was closely related to subsequent delirium; at the same time, individualized blood pressure ranges were obtained. Although, at present, DCA may not be used as an indicator to predict the occurrence of early POD in elderly patients, its potential role in the pathogenesis of POD still deserves attention.

This study identified 5 elderly patients with POD, all of whom were over 70 years old. The average headdown time for these patients exceeded 3 hours. Two of the patients were not included in the DCA analysis due to a significant increase in $\mathrm{P}_{\mathrm{ET}} \mathrm{CO}_{2}$ during surgery, which significantly affected CA. Anesthesiologists should attach importance to the management of intraoperative anesthesia in elderly patients, especially blood pressure management, in order to reduce intraoperative fluctuations in blood pressure and DCA, and to strictly control $\mathrm{CO}_{2}$ absorption, thus appropriately reducing pneumoperitoneal pressure and shortening the length of time spent in the head-down position.
Most patients in this study presented with a typical U-shaped curve, which could be used to retrospectively detect the intraoperative $\mathrm{MAP}_{\mathrm{OPT}}$ and blood pressure range of the middle-aged and elderly patients. There were no statistical differences in the upper or lower limit of blood pressure between the 2 groups; however, the range of autoregulation in the elderly group was significantly narrower than that in the middle-aged group $(\mathrm{P}<0.01)$, which is consistent with the physiological characteristics of older people. An extremely promising clinical application for monitoring CA is to obtain the optimal blood pressure range of patients during surgery, and to provide more accurate and intuitive physiological indicators for brain protection to guide the management of intraoperative anesthesia and avoid a one-size-fits-all blood pressure management strategy (for instance, some ICU guidelines recommend maintaining MAP $\geq 60 \mathrm{mmHg}$ ). Early intraoperative interventions to alleviate DCA may be represent a key approach to improving postoperative neurocognitive complications in high-risk elderly patients (20).

Astrocyte-derived protein $(\mathrm{S} 100 \beta)$ is a marker protein of glial cells which is positively correlated with glial cell damage. NSE is a neuronal marker enzyme, which serves as an important indicator for assessing brain injury and is positively correlated with neuronal injury. The combined detection of the brain injury markers $S 100 \beta$ protein and NSE enzyme can be used in the early diagnosis of brain injury, as well as the assessment of brain injury degree and prognosis. The higher the serum S100 $\beta$ and NSE concentrations, the worse the prognosis, especially in respect to central nervous system complications (21). In the current study, we observed no significant changes in the pre- and postoperative levels of serum S100 $\beta$, NSE, and IL-6 in either group of patients. This result may have resulted from the short blood sampling interval, or it may be related to the length of surgery. The Trendelenburg position duration for most robotic prostate surgeries in our hospital is about 2 hours, which is significantly shorter than that for laparoscopic surgery previously. Roboticassisted laparoscopic in situ ileal replacement of the bladder and other operative procedures require a longer headdown duration, which has a greater impact on the brain physiology of elderly patients; therefore, the intraoperative management of anesthesia deserves more attention (22).

\section{Conclusions}

This study has shown that correlation analysis of $\mathrm{rScO}_{2}$ 
monitored by NIRS and the synchronized invasive MAP can be used to obtain the dynamic $\mathrm{CO}_{\mathrm{X}}$, which reflects the automatic regulation of cerebral blood flow. Taking a $\mathrm{CO}_{\mathrm{X}}$ threshold of $\geq 0.3$ to define DCA, our study revealed that the cumulative time of DCA during surgery is significantly longer in elderly patients than in middle-aged patients. At the same time, the typical U-shape of the $\mathrm{CO}_{\mathrm{X}}$ curve can be applied to obtain the intraoperative $\mathrm{MAP}_{\mathrm{OPT}}$ and optimal blood pressure range, which may facilitate individualized and precise intraoperative blood pressure management, thus improving postoperative neurocognition in high-risk elderly patients.

\section{Acknowledgments}

Funding: This work was supported by the National Key R\&D Program of China (No. 2018YFC2001901) and Project for Clinical Research of the PLA General Hospital (No. 2018XXFC-8, No. 2019XXJSYX08, and No. 2019XXMBD-003).

\section{Footnote}

Reporting Checklist: The authors have completed the TREND reporting checklist. Available at http://dx.doi. org/10.21037/apm-21-707

Data Sharing Statement: Available at http://dx.doi. org/10.21037/apm-21-707

Conflicts of Interest: All authors have completed the ICMJE uniform disclosure form (available at http://dx.doi. org/10.21037/apm-21-707). All authors report that this work was supported by the National Key R\&D Program of China (No. 2018YFC2001901) and Project for Clinical Research of the PLA General Hospital (No. 2018XXFC-8, No. 2019XXJSYX08, and No. 2019XXMBD-003).

Ethical Statement: The authors are accountable for all aspects of the work in ensuring that questions related to the accuracy or integrity of any part of the work are appropriately investigated and resolved. This study was reviewed and approved by the Ethics Committee of the PLA General Hospital (No. S2019-120-02). A signed informed consent form was obtained from each patient or from a member of their family. All procedures performed in this study involving human participants were in accordance with the Declaration of Helsinki (as revised in 2013).
Open Access Statement: This is an Open Access article distributed in accordance with the Creative Commons Attribution-NonCommercial-NoDerivs 4.0 International License (CC BY-NC-ND 4.0), which permits the noncommercial replication and distribution of the article with the strict proviso that no changes or edits are made and the original work is properly cited (including links to both the formal publication through the relevant DOI and the license). See: https://creativecommons.org/licenses/by-nc-nd/4.0/.

\section{References}

1. Mikhail D, Sarcona J, Mekhail M, et al. Urologic Robotic Surgery. Surg Clin North Am 2020;100:361-78.

2. Kalmar AF, Dewaele F, Foubert L, et al. Cerebral haemodynamic physiology during steep Trendelenburg position and $\mathrm{CO}_{2}$ pneumoperitoneum. Br J Anaesth 2012;108:478-84.

3. Gainsburg DM. Anesthetic concerns for robotic-assisted laparoscopic radical prostatectomy. Minerva Anestesiol 2012;78:596-604.

4. Marcantonio ER. Delirium in Hospitalized Older Adults. N Engl J Med 2017;377:1456-66.

5. Oh ES, Fong TG, Hshieh TT, et al. Delirium in Older Persons: Advances in Diagnosis and Treatment. JAMA 2017;318:1161-74.

6. Daiello LA, Racine AM, Yun Gou R, et al. Postoperative Delirium and Postoperative Cognitive Dysfunction. Anesthesiology 2019;131:477-91.

7. Ono M, Brady K, Easley RB, et al. Duration and magnitude of blood pressure below cerebral autoregulation threshold during cardiopulmonary bypass is associated with major morbidity and operative mortality. J Thorac Cardiovasc Surg 2014;147:483-9.

8. Hempstead KA, Phillips JA. Rising suicide among adults aged 40-64 years: the role of job and financial circumstances. Am J Prev Med 2015;48:491-500.

9. Burkhart CS, Rossi A, Dell-Kuster S, et al. Effect of age on intraoperative cerebrovascular autoregulation and nearinfrared spectroscopy-derived cerebral oxygenation. Br J Anaesth 2011;107:742-8.

10. Moerman A, De Hert S. Recent advances in cerebral oximetry. Assessment of cerebral autoregulation with near-infrared spectroscopy: myth or reality? F1000Res 2017;6:1615.

11. Jöbsis FF. Noninvasive, infrared monitoring of cerebral and myocardial oxygen sufficiency and circulatory parameters. Science 1977;198:1264-7. 
12. Sakudo A. Near-infrared spectroscopy for medical applications: Current status and future perspectives. Clin Chim Acta 2016;455:181-8.

13. Serraino GF, Murphy GJ. Effects of cerebral near-infrared spectroscopy on the outcome of patients undergoing cardiac surgery: a systematic review of randomised trials. BMJ Open 2017;7:e016613.

14. Rogers CA, Stoica S, Ellis L, et al. Randomized trial of near-infrared spectroscopy for personalized optimization of cerebral tissue oxygenation during cardiac surgery. Br J Anaesth 2017;119:384-93.

15. Vranken NPA, Weerwind PW, Sutedja NA, et al. Cerebral Oximetry and Autoregulation during Cardiopulmonary Bypass: A Review. J Extra Corpor Technol 2017;49:182-91.

16. Goettel N, Burkhart CS, Rossi A, et al. Associations Between Impaired Cerebral Blood Flow Autoregulation, Cerebral Oxygenation, and Biomarkers of Brain Injury and Postoperative Cognitive Dysfunction in Elderly Patients After Major Noncardiac Surgery. Anesth Analg 2017;124:934-42.

17. Ono M, Joshi B, Brady K, et al. Risks for impaired cerebral autoregulation during cardiopulmonary bypass and

Cite this article as: Zhang Y, Tan J, Li P, Zhang X, Yang Y, Liu Y, Fu Q, Cao J, Mi W, Zhang H, Li H. The perioperative application of continuous cerebral autoregulation monitoring for cerebral protection in elderly patients. Ann Palliat Med 2021;10(4):4582-4592. doi: 10.21037/apm-21-707 postoperative stroke. Br J Anaesth 2012;109:391-8.

18. Hori D, Brown C, Ono M, et al. Arterial pressure above the upper cerebral autoregulation limit during cardiopulmonary bypass is associated with postoperative delirium. Br J Anaesth 2014;113:1009-17.

19. Lee KF WM, Maslove DM, et al. Dysfunctional cerebral autoregulation is associated with delirium in critically ill middle-ageds. J Cereb Blood Flow Metab 2019;39:2512-20.

20. Denault AY, Brassard P, Jacquet-Lagreze M, et al. Targeting optimal blood pressure monitoring: what's next? J Thorac Dis 2018;10:S3281-5.

21. Yu Y, Han RQ. The application of cerebral biomarkers in traumatic craniocerebral injury. International Journal of Anesthesiology and Resuscitation 2013;34:126-9.

22. Pandey R, Garg R, Darlong V, et al. Unpredicted neurological complications after robotic laparoscopic radical cystectomy and ileal conduit formation in steep trendelenburg position: two case reports. Acta Anaesthesiol Belg 2010;61:163-6.

(English Language Editor: J. Reynolds) 